\title{
A NEW LINEARLY EXTRAPOLATED CRANK-NICOLSON TIME-STEPPING SCHEME FOR THE NAVIER-STOKES EQUATIONS
}

\author{
ROSS INGRAM
}

\begin{abstract}
We investigate the stability of a fully-implicit, linearly extrapolated Crank-Nicolson (CNLE) time-stepping scheme for finite element spatial discretization of the Navier-Stokes equations. Although presented in 1976 by Baker and applied and analyzed in various contexts since then, all known convergence estimates of CNLE require a time-step restriction. We propose a new linear extrapolation of the convecting velocity for CNLE that ensures energetic stability without introducing an undesirable exponential Gronwall constant. Such a result is unknown for conventional CNLE for inhomogeneous boundary data (usual techniques fail!). Numerical illustrations are provided showing that our new extrapolation clearly improves upon stability and accuracy from conventional CNLE.
\end{abstract}

\section{INTRODUCTION}

The Navier-Stokes (NS) equations (NSE) provide an accurate description of fluid flow. However, there are many subtle and unresolved questions regarding existence and smoothness of the NS velocity field $\mathbf{u}$. There are related open questions regarding the development and implementation of stable, accurate, robust, and feasible methods for approximating $\mathbf{u}$. Suppressing spatial discretization, the usual, linearly implicit Crank-Nicolson (CN) method (also called CNLE-CN with Linear Extrapolation) for the NSE is: given $\mathbf{u}^{0}, \mathbf{u}^{1}$, and $p^{1}$, for each $n=1,2, \ldots$ find velocity $\mathbf{u}^{n+1}$ and pressure $p^{n+1}$ satisfying

$$
\begin{aligned}
& \text { (1) } \frac{\mathbf{u}^{n+1}-\mathbf{u}^{n}}{\Delta t}+\left(\frac{3}{2} \mathbf{u}^{n}-\frac{1}{2} \mathbf{u}^{n-1}\right) \cdot \nabla \mathbf{u}^{n+1 / 2}-\nu \Delta \mathbf{u}^{n+1 / 2}+\nabla p^{n+1 / 2}=\mathbf{f}^{n+1 / 2}, \\
& \text { (2) } \nabla \cdot \mathbf{u}^{n+1}=0 .
\end{aligned}
$$

Here $\Delta t>0$ is the time-step, $\mathbf{f}$ is body-force term, $\nu>0$ the kinematic viscosity of the fluid, and $z^{n+1 / 2}=\frac{1}{2}\left(z^{n+1}+z^{n}\right)$. Equations (11), (2) have been widely studied since proposed and analyzed by Baker in [2, e.g., [3, 6, 17, 20, 25. Let $\Omega \subset \mathbb{R}^{d}$ for $d=2$ or 3 be the problem domain. CNLE is generally believed to be comparable in stability and accuracy to the more expensive, fully implicit, nonlinear CN method denoted CN-NSE. We show that this is not the case for problems with

Received by the editor June 14, 2011 and, in revised form, January 8, 2012.

2010 Mathematics Subject Classification. Primary 65M12, 65M60, 76D05, 76M10, 76M20; Secondary 76D15, 76M25, 65M22.

Key words and phrases. Navier-Stokes, Crank-Nicolson, finite element, extrapolation, linearization, implicit, stability, analysis, inhomogeneous.

The author was partially supported by NSF Grants DMS 0508260 and 080385. 
inhomogeneous boundary data

$$
\left.\mathbf{u}\right|_{\partial \Omega}=\phi \neq 0
$$

such as simple channel flow with inflow-outflow boundaries. Additionally, we derive a new, linearly implicit variation of $\mathrm{CN}$ that corrects for the subtle problems associated with solutions to (1), (2) under (3).

CN-NSE is well-known to be unconditionally nonlinearly (energetically) stable; see e.g. [19] and references therein. We show, however, that within current techniques, the standard $\mathcal{O}\left(\Delta t^{2}\right)$ linear extrapolation in (1) does not lead to a (provable) energetically stable numerical discretization in the case of inhomogeneous problem data for long-time solutions. Specifically, stability has not been proven and known methods of proof fail. We propose a new $\mathcal{O}\left(\Delta t^{2}\right)$ extrapolation for general data:

$$
\frac{\mathbf{u}^{n+1}+\mathbf{u}^{n}}{2} \cdot \nabla \mathbf{u} \approx \xi^{n}(\mathbf{u}) \cdot \nabla \mathbf{u}, \quad \xi^{n}(\mathbf{u}):=2\left(\frac{\mathbf{u}^{n}+\mathbf{u}^{n-1}}{2}\right)-\frac{\mathbf{u}^{n-1}+\mathbf{u}^{n-2}}{2} .
$$

Note the increased stencil requires additional storage (i.e., $\mathbf{u}^{n}, \mathbf{u}^{n-1}, \mathbf{u}^{n-2}$ at each time-step) compared to conventional CNLE (that requires only $\mathbf{u}^{n}, \mathbf{u}^{n-1}$ ). We show herein that CNLE approximations $\left\{\mathbf{u}^{n}\right\}_{n}$ obtained with (4) are provably stable for general data (3) so that

$$
\max _{n}\left\|\mathbf{u}^{n+1}\right\|^{2}+\nu \Delta t \sum_{n}\left\|\nabla \mathbf{u}^{n+1 / 2}\right\|^{2} \leq C(\text { data })<\infty .
$$

It is illuminating to introduce the backward-Euler (BE) scheme (stable for general data) to highlight the difficulties of inhomogeneous CNLE. First, the stability analysis for homogeneous data relies on the skew-symmetry of the convective nonlinearity in the NSE:

$$
\left.\mathbf{u}\right|_{\partial \Omega}=0, \quad \nabla \cdot \mathbf{u}=0 \Rightarrow \int_{\Omega} \mathbf{u} \cdot \nabla \mathbf{u} \cdot \mathbf{u}=0 .
$$

Let $i=1$ for BE with linear extrapolation (BELE) and $i=2$ for CNLE. The energy difference due to the numerical extrapolation

(5) $\int \mathbf{u}^{n+1 / i} \cdot \nabla \mathbf{u}^{n+1 / i} \cdot \mathbf{v} \approx \int \xi^{n}(\mathbf{u}) \cdot \nabla \mathbf{u}^{n+1 / i} \cdot \mathbf{v}, \quad \xi^{n}(\mathbf{u}):=a_{0} \mathbf{u}^{n}+\ldots+a_{n_{0}} \mathbf{u}^{n-n_{0}}$

must be absorbed into the model viscous term $\nu \sum_{n}\left\|\nabla \mathbf{u}^{n+1 / i}\right\|^{2}$ to establish energetic stability for $T \rightarrow \infty$. Indeed, we lift the data with an extension operator $E(\phi)$ so that

$$
\mathbf{u}=\mathbf{u}_{0}+E(\phi),\left.\quad \mathbf{u}_{0}\right|_{\partial \Omega}=0,\left.\quad E(\phi)\right|_{\partial \Omega}=\phi .
$$

Cross-terms from the nonlinearity pollute the RHS of the resulting estimate upon the substitution $\mathbf{u}^{n}=\mathbf{u}_{0}^{n}+E\left(\phi^{n}\right)$. The energy estimate for $\mathbf{u}_{0}^{n}$ is obtained by testing either BELE or CNLE with $\mathbf{v}=\mathbf{u}_{0}^{n+1 / i}$ to get

$$
\begin{aligned}
& \left\|\mathbf{u}_{0}^{n+1}\right\|^{2}+\nu \Delta t \sum_{n}\left\|\nabla \mathbf{u}_{0}^{n+1 / i}\right\|^{2}+\ldots \\
& \quad=-\Delta t \sum_{n} \int \xi^{n}\left(\mathbf{u}_{0}\right) \cdot \nabla E\left(\phi^{n+1 / i}\right) \cdot \mathbf{u}_{0}^{n+1 / i}+\ldots
\end{aligned}
$$

Suppose that the extension $E(\phi)$ satisfies

$$
\left|\int \xi^{n}\left(\mathbf{u}_{0}\right) \cdot \nabla E\left(\phi^{n+1 / i}\right) \cdot \mathbf{u}_{0}^{n+1 / i}\right| \leq \delta\left\|\nabla \xi^{n}\left(\mathbf{u}_{0}\right)\right\|\left\|\nabla \mathbf{u}_{0}^{n+1 / i}\right\|
$$


for some $\delta>0$. In the continuous-space case, for each $\delta>0$, there exists $E(\phi)$ satisfying (77). Suppose that $\xi^{n}(\mathbf{u})=\mathbf{u}^{n}$ for BELE and $\xi^{n}(\mathbf{u})=\frac{3}{2} \mathbf{u}^{n}-\frac{1}{2} \mathbf{u}^{n-1}$ for CNLE. We apply (7) to derive an a priori estimate for $\mathbf{u}_{0}$ from (6). One option is to bound the right-hand side of (7) so that

$$
\begin{aligned}
& \left|\int \xi^{n}\left(\mathbf{u}_{0}\right) \cdot \nabla E\left(\phi^{n+1 / i}\right) \cdot \mathbf{u}_{0}^{n+1 / i}\right| \\
& \quad \leq \frac{\delta}{2} \begin{cases}\left(\left\|\nabla \mathbf{u}_{0}^{n+1}\right\|^{2}+\left\|\nabla \mathbf{u}_{0}^{n}\right\|^{2}\right), & \text { BELE, } \\
\left(\left\|\nabla\left(\frac{3}{2} \mathbf{u}_{0}^{n}-\frac{1}{2} \mathbf{u}_{0}^{n-1}\right)\right\|^{2}+\left\|\nabla \mathbf{u}_{0}^{n+1 / 2}\right\|^{2}\right), & \text { CNLE. }\end{cases}
\end{aligned}
$$

We can absorb $\frac{\delta}{2} \sum_{n}\left(\left\|\nabla \mathbf{u}_{0}^{n+1}\right\|^{2}+\left\|\nabla \mathbf{u}_{0}^{n}\right\|^{2}\right)$ into $\nu \sum_{n}\left\|\nabla \mathbf{u}_{0}^{n+1}\right\|^{2}$ in (6) for BELE. However, regardless of how small $\delta$ is taken, there is no way in general to absorb $\frac{\delta}{2} \sum_{n}\left\|\nabla\left(\frac{3}{2} \mathbf{u}_{0}^{n}-\frac{1}{2} \mathbf{u}_{0}^{n-1}\right)\right\|^{2}$ into $\nu \sum_{n}\left\|\nabla \mathbf{u}_{0}^{n+1 / 2}\right\|^{2}$ in (6) for CNLE. Indeed, in the extreme case that $\mathbf{v}^{n}=-\mathbf{v}^{n+1} \neq 0$, then $\left\|\nabla \mathbf{v}^{n+1 / 2}\right\|^{2}=0$ while $\left\|\nabla \mathbf{v}^{n}\right\|^{2}>0$ so that no small data restriction on $\nu$ or $\phi \neq 0$ will help absorb the latter into the former. Instead, we restrict linearizations (5) to satisfy (4). Extrapolation (4) allows the RHS of (8) (CNLE) to be replaced by $\left\|\nabla \mathbf{u}_{0}^{n+1 / 2}\right\|^{2}+\sum_{i=1}^{2}\left\|\nabla \mathbf{u}_{0}^{n-i+1 / 2}\right\|^{2}$. For small enough $\delta>0$, we can now absorb $\frac{\delta}{2} \sum_{n}\left(\left\|\nabla \mathbf{u}_{0}^{n+1 / 2}\right\|^{2}+\sum_{i=1}^{2}\left\|\nabla \mathbf{u}_{0}^{n-i+1 / 2}\right\|^{2}\right)$ into $\nu \sum_{n}\left\|\nabla \mathbf{u}_{0}^{n+1 / 2}\right\|^{2}$ in (6).

A discrete Gronwall lemma can be applied instead of (7), but introduces the factor

$$
C(\text { data }) \propto \exp \left(\nu^{q-1} \sum_{n}\left\|E\left(\phi^{n}\right)\right\|_{W^{q, \infty}}^{2-q}\right), \quad q=0, \text { or } 1
$$

so that the a priori estimate of CNLE solutions in the energy norm grows exponentially with problem data and $T$. Ultimately the Gronwall constant gives very poor long-time estimates and, to preserve the applicability of a numerical method, should be avoided for a priori energy estimates.

We provide a brief overview of extrapolation schemes for CN-NSE with references in Section 1.1. We formulate the continuous and discrete setting for analysis in Sections 1.2, 1.3. We consider finite element (FE) spatial discretization in conjunction with time-stepping for BE (BE-FEM) and CN (CN-FEM). In Section 2 we present and prove stability of BELE and CNLE (with extrapolations of the form (4)) for inhomogeneous data. In Section 3, we conclude with a numerical investigation in which we compare CN-FEM (with Newton nonlinear iterations), traditional CNLE in (1), and CNLE with extrapolation (4) denoted CNLE(stab). For flow past a 2d cylinder, for a given time-step, the energy dissipation rate for CNLE(stab) approximations more closely matches CN-FEM (with Newton) than CNLE. In fact, for a given time-step, CNLE fails to predict the vortex shedding in the wake of the cylinder (overly diffusive) whereas CNLE(stab) captures the physics properly.

1.1. Motivation of fully implicit linearizations of the NSE. A central question in practical computational fluid dynamics concerns the smallest amount of work permitted to produce a stable and accurate approximation of the flow field. The method for approximating NS fluid flows is largely influenced by the following:

- stiffness of problem in diffusion-dominated flow regions,

- lack of and/or unknown regularity of true NS-solution,

- large $R e \Rightarrow$ many mesh points $\Rightarrow$ extremely large system of DAE's.

(where DAE's are Differential Algebraic Equations). Implicit time-stepping approximations of the NSE are preferred in practice in order to avoid unnecessary 
numerical/modeling restrictions on the time-step size. We investigate the stability and accuracy of a linearly extrapolated version of the CN time-stepping scheme for the NSE which eliminates the necessity of multiple, time-intensive, nonlinear iterations at each time-step. Adaptive time-stepping techniques can (and should!) be applied in conjunction with any time-discretization to (significantly) reduce computational costs.

There are many analyses of CN time-stepping methods for the NSE. Heywood and Rannacher 19 provide analysis of CN-FEM. The 2nd and 3rd order CNLE methods are introduced and analyzed in [2, 3. Multilevel methods based on CNLE (building on the work in [26] and [10]) are analyzed in [17, 20. CNLE approximation of a stochastic NSE is analyzed in [6]. The authors in [25] analyze a stabilized CNLE method. Each of these analyses requires, explicitly or implicitly stated, a time-step restriction of the form

$$
\Delta t \leq C(R e, h)
$$

to guarantee convergence. A 1st order CNLE is used in 22 in conjunction with coupled multigrid and pressure Schur complement schemes for the NSE. Numerical comparison of various NS time-stepping schemes (excluding CNLE) are provided in [24]. A CN/Adams-Bashforth (CN-AB) time-stepping scheme is another linear variant of CN-FEM. Unlike CNLE, CN-AB is explicit in the nonlinearity and only conditionally stable [16] (i.e., a time-step restriction of form (9) is required for stability). CN-AB is a popular method for approximating NS flows because it is fast and easy to implement. Each time-step requires only one discrete Stokes system and linear solve. For example, it is used to model turbulent flows induced by wind turbine motion [33, turbulent flows transporting particles in [28], and reacting flows in complex geometries (e.g. gas turbine combustors) [1. The CN method is also applied, for example, to a general class of nonstationary partial differential equations encompassing reaction-diffusion type equations including the nonlinear Sobolev equations [29] and the Ginzburg-Landau model [21. Time-step restrictions of type (9) (where $R e$ has a different meaning) are implicitly required in the convergence analyses of these discrete models.

Error estimates for BE time-stepping is analyzed in [11 (semi-discrete) and [34] (fully-discrete). Although the most stable time-stepping scheme, BE methods are only $\Delta t$-accurate. Higher order backward difference methods like BDF2 are considered the best choice in general for time-stepping (more stable than $\mathrm{CN}$ and $\Delta t^{2}$-accurate), but introduce artificial dissipation which is avoided by CN methods. See [13] (e.g. Chapter 3.16) for an overview of the analysis and treatment of many time-stepping schemes available for approximating NS-flows with a welldocumented discussion of the advantages and disadvantages of each method.

1.2. Continuous function setting. Let $\mathbf{a}:=\left(a_{0}, a_{1}, \ldots, a_{n_{0}}\right) \in \mathbb{R}^{n_{0}+1}$ for some $n_{0} \in\{0\} \cup \mathbb{N}$ be equipped with the standard $l^{q}$ norm denoted by $|\mathbf{a}|_{q}$ for $1 \leq q \leq \infty$. Fix $p \geq 1$. Let $L^{p}(\Omega)$ denote the linear space of all real Lebesgue-measurable functions and bounded in the usual norm denoted by $\|\cdot\|_{L^{p}(\Omega)}$. Let $(\cdot, \cdot)_{\Omega}$ and $\|\cdot\|_{\Omega}$ be the standard $L^{2}(\Omega)$-inner product and norm. Fix $k \in \mathbb{R}$. The Sobolev space $W^{k, p}(\Omega)$ is equipped with the usual norm denoted by $\|\mathbf{u}\|_{W^{k, p}(\Omega)}$. Identify $\|\cdot\|_{k, p, \Omega}:=\|\cdot\|_{W^{k, p}(\Omega)}, H^{k}(\Omega):=W^{k, 2}(\Omega)$ and $\|\cdot\|_{k, \Omega}:=\|\cdot\|_{W^{k, 2}(\Omega)}$ with $|\cdot|_{k, \Omega}$ the corresponding semi-norm. Let the context determine whether $W^{k, p}(\Omega)$ denotes 
a scalar, vector, or tensor function space. For example, let $\mathbf{v}: \Omega \rightarrow \mathbb{R}^{d}$. Then, $\mathbf{v} \in H^{1}(\Omega)$ implies that $\mathbf{v} \in H^{1}(\Omega)^{d}$ and $\nabla \mathbf{v} \in H^{1}(\Omega)$ implies that $\nabla \mathbf{v} \in H^{1}(\Omega)^{d \times d}$.

Fix $\phi \in H^{1 / 2}(\partial \Omega)$ (an element of the trace of $H^{1}(\Omega)$ functions) satisfying $\int_{\partial \Omega} \phi$. $\hat{\mathbf{n}}=0$ where $\hat{\mathbf{n}}$ is the outward (relative to $\Omega$ ) unit normal defined a.e. on $\partial \Omega$. Define

$$
H_{\phi}^{1}(\Omega):=\left\{\mathbf{v} \in H^{1}(\Omega):\left.\mathbf{v}\right|_{\partial \Omega}=\phi\right\}, \quad V_{\phi}(\Omega):=\left\{\mathbf{v} \in H_{\phi}^{1}(\Omega): \nabla \cdot \mathbf{v}=0\right\} .
$$

Write $V(\Omega)=V_{0}(\Omega)$. Moreover, the dual space of $H_{0}^{1}(\Omega)$ is denoted $W^{-1,2}(\Omega):=$ $\left(H_{0}^{1}(\Omega)\right)^{\prime}$ and equipped with the norm

$$
\|\mathbf{f}\|_{-1, \Omega}:=\sup _{0 \neq \mathbf{v} \in H_{0}^{1}(\Omega)} \frac{\langle\mathbf{f}, \mathbf{v}\rangle_{W^{-1,2}(\Omega) \times H_{0}^{1}(\Omega)}}{|\mathbf{v}|_{1, \Omega}} .
$$

Define

$$
L_{0}^{2}(\Omega):=\left\{q \in L^{2}(\Omega):(q, 1)=0\right\} .
$$

For brevity, omit $\Omega$ in the definitions above. For example, $(\cdot, \cdot)=(\cdot, \cdot)_{\Omega}, H^{1}=$ $H^{1}(\Omega)$, and $V=V_{0}(\Omega)$. It is convenient in the analysis of problems with inhomogeneous data to introduce the following function spaces:

$$
V_{*}:=\left\{\mathbf{v} \in H^{1}: \nabla \cdot \mathbf{v}=0\right\}, \quad H_{0}^{1 / 2}(\partial \Omega):=\left\{\mu \in H^{1 / 2}(\partial \Omega): \int_{\partial \Omega} \mu \cdot \hat{\mathbf{n}}=0\right\} .
$$

There exists an extension operator $E: H_{0}^{1 / 2}(\partial \Omega) \rightarrow V_{*}$ (see e.g. [9], pp. 131-132). Note that all such extensions satisfy $E(\mathbf{0}) \in V$.

Fix time $T>0$ and $m \geq 1$. Let $W^{m, q}\left(0, T ; W^{k, p}(\Omega)\right)$ denote the linear space of all Lebesgue measurable functions from $(0, T)$ onto $W^{k, p}$ equipped with and bounded in the norm

$$
\|\mathbf{u}\|_{W^{m, q}\left(0, T ; W^{k, p}\right)}:=\left(\int_{0}^{T} \sum_{i=0}^{m}\left\|\partial_{t}^{(i)} \mathbf{u}(\cdot, t)\right\|_{W^{k, p}}^{q} d t\right)^{1 / q} .
$$

Write $W^{m, q}\left(W^{k, p}\right)=W^{m, q}\left(0, T ; W^{k, p}(\Omega)\right)$ and $C^{m}\left(W^{k, p}\right)=C^{m}\left([0, T] ; W^{k, p}(\Omega)\right)$.

1.3. Discrete function setting. Fix $h>0$. Let $\mathcal{T}_{h}$ be a family of subdivisions (e.g. triangulation) of $\bar{\Omega} \subset \mathbb{R}^{d}$ satisfying $\bar{\Omega}=\bigcup_{E \in \mathcal{T}_{h}} E$ so that diameter $(E) \leq h$ and any two closed elements $E_{1}, E_{2} \in \mathcal{T}_{h}$ are either disjoint or share exactly one face, side, or vertex. See Chapter II, Appendix A in [12] for more on this subject in context of Stokes problem and [5] for a more general treatment. For example, $\mathcal{T}_{h}$ consists of triangles for $d=2$ or tetrahedra for $d=3$ that are nondegenerate as $h \rightarrow 0$.

Let $X_{h, *} \subset\left(H^{1}\right)^{d}$ and $Q_{h, *} \subset L^{2}$ be a mixed finite element (FE) space. For example, let $X_{h, *}$ and $Q_{h, *}$ be continuous, piecewise (on each $E \in \mathcal{T}_{h}$ ) polynomial spaces. Fix $\phi_{h} \approx \phi$ so that there exists $\mathbf{v} \in X_{h, *}$ satisfying $\left.\mathbf{v}\right|_{\partial \Omega}=\phi_{h}$. Define $X_{h, \phi_{h}}:=X_{h, *} \cap H_{\phi_{h}}^{1}, Q_{h}:=Q_{h, *} \cap L_{0}^{2}$. The discretely divergence-free space is given by

$$
V_{h, \phi_{h}}=\left\{\mathbf{v}_{h} \in X_{h, \phi_{h}}:\left(q_{h}, \nabla \cdot \mathbf{v}_{h}\right)=0 \quad \forall q_{h} \in Q_{h, *}\right\} .
$$

Write $V_{h}=V_{h, 0}, X_{h}=X_{h, 0}$. Note that in general $V_{h} \not \subset V$ (e.g. Taylor-Hood elements). Define the discrete trace space of $X_{h}$ by

$$
\begin{aligned}
& \Lambda_{h}(\partial \Omega):=\left\{\lambda_{h}: H^{1 / 2}(\partial \Omega): \exists \mathbf{v}_{h} \in X_{h, *}\right. \text { such that } \\
& \left.\left.\lambda_{h}\right|_{\partial E \cap \partial \Omega}=\left.\mathbf{v}_{h}\right|_{\partial E \cap \partial \Omega} \forall E \in \mathcal{T}_{h} \text { and }|\partial E \cap \partial \Omega|>0\right\} .
\end{aligned}
$$


Next define discrete analogues to $V_{*}$ and $H_{0}^{1 / 2}(\partial \Omega)$ respectively by

$$
\begin{aligned}
V_{h, *}: & =\left\{\mathbf{v}_{h} \in X_{h, *}:\left(q_{h}, \nabla \cdot \mathbf{v}_{h}\right)=0 \forall q_{h} \in Q_{h, *}\right\} \\
\Lambda_{h, 0}(\partial \Omega): & =\left\{\mu_{h} \in \Lambda_{h}(\partial \Omega): \int_{\partial \Omega} \mu_{h} \cdot \hat{\mathbf{n}}=0\right\} .
\end{aligned}
$$

Then there exists a discrete extension operator $E_{h}: \Lambda_{h, 0}(\partial \Omega) \rightarrow V_{h, *}$ (see e.g. [4, 14, 32]). Note that all such extensions satisfy $E_{h}(\mathbf{0}) \in V_{h}$.

We assume that $X_{h} \times Q_{h}$ satisfies the uniform inf-sup (LBB) condition:

$$
\inf _{q_{h} \in Q_{h}} \sup _{\mathbf{v}_{h} \in X_{h}} \frac{\left(q_{h}, \nabla \cdot \mathbf{v}_{h}\right)}{\left|\mathbf{v}_{h}\right|_{1}|| q||} \geq C>0
$$

where $C$ is independent of $h \rightarrow 0$. The well-known Taylor-Hood element is one such example satisfying (10).

Set $0=t^{0}<t^{1}<\ldots<t^{n}=T<\infty$ with constant time-step $\Delta t=t^{n}-t^{n-1}$. Write $z^{n}:=z\left(t^{n}\right)$ and $z^{n+1 / 2}:=\frac{1}{2}\left(z\left(t^{n+1}\right)+z\left(t^{n}\right)\right)$. Define

$$
\|\mathbf{u}\|_{l^{q}\left(m_{1}, m_{2} ; W^{k, p}\right)}:= \begin{cases}\left(\Delta t \sum_{n=m_{1}}^{m_{2}}\left\|\mathbf{u}^{n}\right\|_{k, p}^{q}\right)^{1 / q}, & q \in[1, \infty), \\ \max _{m_{1} \leq n \leq m_{2}}\left\|\mathbf{u}^{n}\right\|_{k, p}, & q=\infty,\end{cases}
$$

for any $0 \leq n=m_{1}, m_{1}+1, \ldots, m_{2} \leq N$. Write $\|\mathbf{u}\|_{l^{q}\left(W^{k, p}\right)}=\|\mathbf{u}\|_{l^{q}\left(0, N ; W^{k, p}\right)}$. We say that $\mathbf{u} \in l^{q}\left(m_{1}, m_{2} ; W^{k, p}\right)$ is bounded in respective norm as $\Delta t \rightarrow 0$. Define the discrete time-derivative

$$
\partial_{\Delta t}^{n+1} \mathbf{v}:=\frac{\mathbf{v}^{n+1}-\mathbf{v}^{n}}{\Delta t}
$$

In order to avoid stability issues arising when FE solutions are not exactly divergence free (i.e., when $V_{h} \not \subset V$ ), we introduce the explicitly skew-symmetric convective term

$$
c_{h}(\mathbf{u}, \mathbf{v}, \mathbf{w}):=\frac{1}{2}((\mathbf{u} \cdot \nabla \mathbf{v}, \mathbf{w})-(\mathbf{u} \cdot \nabla \mathbf{w}, \mathbf{v}))
$$

so that

$$
c_{h}(\mathbf{u}, \mathbf{v}, \mathbf{v})=0
$$

Fix $a_{i} \in \mathbb{R}$ for $i=0,1, \ldots, n_{0} \geq 0$ and $n \in\{0\} \cup \mathbb{N}$. Define the linearization operator $\xi^{n}(\mathbf{u})$ so that

$$
c_{h}(\mathbf{u}, \mathbf{v}, \mathbf{w}) \approx c_{h}\left(\xi^{n}(\mathbf{u}), \mathbf{v}, \mathbf{w}\right), \quad \xi^{n}(\mathbf{u}):=a_{-1} \mathbf{u}^{n+1}+a_{0} \mathbf{u}^{n}+\ldots+a_{n_{0}} \mathbf{u}^{n-n_{0}} .
$$

For example,

$$
\begin{array}{lll}
\xi^{n}(\mathbf{u})=\frac{1}{2}\left(3 \mathbf{u}^{n}-\mathbf{u}^{n-1}\right) & \Rightarrow & \xi^{n}(\mathbf{u})=\mathbf{u}\left(\cdot, t^{n+1 / 2}\right)+\mathcal{O}\left(\Delta t^{2}\right) \\
\xi^{n}(\mathbf{u})=2 \mathbf{u}^{n-1 / 2}-\mathbf{u}^{n-3 / 2} & \Rightarrow & \xi^{n}(\mathbf{u})=\mathbf{u}\left(\cdot, t^{n+1 / 2}\right)+\mathcal{O}\left(\Delta t^{2}\right) .
\end{array}
$$

Note that $a_{-1}=0$ gives a linear NSE approxmation and $a_{-1} \neq 0$ gives a nonlinear (fully implicit) NSE approximation. 


\section{Stable linearizations When $\left.\mathbf{u}_{h}\right|_{\partial \Omega} \neq 0$}

Fix $\mathbf{f} \in W^{-1,2}$ and $\nu>0$. In this setting, we consider strong NS solutions: find $\mathbf{u} \in L^{2}\left(H_{\phi}^{1}\right) \cap L^{\infty}\left(L^{2}\right)$ and $p \in W^{-1, \infty}\left(L_{0}^{2}\right)$ satisfying

$$
\begin{aligned}
& \left(\partial_{t} \mathbf{u}, \mathbf{v}\right)+(\mathbf{u} \cdot \nabla \mathbf{u}, \mathbf{v})+\nu(\nabla \mathbf{u}, \nabla \mathbf{v})-(p, \nabla \cdot \mathbf{v})=(\mathbf{f}, \mathbf{v}), \quad \forall \mathbf{v} \in H_{0}^{1}, \\
& \nabla \cdot \mathbf{u}(\cdot, t)=0 \quad \text { in } L^{2}, \quad \text { a.e. } t \in(0, T), \\
& \mathbf{u}(\cdot, 0)=\mathbf{u}^{0} \quad \text { in } L^{2} .
\end{aligned}
$$

Next, we pose a FE discretization of (13), (14), (15). BE is the simplest implicit time-stepping scheme with $\Delta t$-accuracy and excellent stability properties.

Problem 2.1 (BELE). Let $\mathbf{u}_{h}^{i} \in V_{h, \phi_{h}^{i}}$ approximate $\mathbf{u}^{i}$ for each $i=0,1, \ldots, n_{0}$. For each $n=n_{0}, n_{0}+1, \ldots, N-1$, find $\left(\mathbf{u}_{h}^{n+1}, p_{h}^{n+1}\right) \in X_{h, \phi_{h}^{n+1}} \times Q_{h}$ satisfying

$$
\begin{aligned}
& \left(\partial_{\Delta t}^{n+1} \mathbf{u}_{h}, \mathbf{v}_{h}\right)+c_{h}\left(\xi^{n}\left(\mathbf{u}_{h}\right), \mathbf{u}_{h}^{n+1}, \mathbf{v}_{h}\right) \\
& \quad+\nu\left(\nabla \mathbf{u}_{h}^{n+1}, \nabla \mathbf{v}_{h}\right)-\left(p_{h}^{n+1}, \nabla \cdot \mathbf{v}_{h}\right)=\left\langle\mathbf{f}^{n+1}, \mathbf{v}_{h}\right\rangle, \quad \forall \mathbf{v}_{h} \in X_{h}, \\
& \quad\left(q_{h}, \nabla \cdot \mathbf{u}^{n+1}\right)=0, \quad \forall q_{h} \in Q_{h} .
\end{aligned}
$$

Remark 2.2. Note that $\xi^{n}\left(\mathbf{u}_{h}\right)=\mathbf{u}_{h}^{n+1}\left(n_{0}=0, a_{-1}=1, a_{0}=0\right)$ defines BE-FEM and $\xi^{n}\left(\mathbf{u}_{h}\right)=\mathbf{u}_{h}^{n}\left(n_{0}=0, a_{-1}=0, a_{0}=1\right)$ defines BELE (see e.g. [11, 13, 18, 34]). Also note that it is possibly desirable to pick $n_{0}>0$ for a better approximation of the lagged convecting velocity.

$\mathrm{CN}$ methods are $\Delta t^{2}$-accurate (more accurate than $\mathrm{BE}$ ), but require consistent initial conditions including pressure. CNLE is a particularly attractive method because it is $\Delta t^{2}$-accurate, implicit in the convective term (a source of stiffness), and linear.

Problem 2.3 (CNLE). Let $\mathbf{u}_{h}^{i} \in V_{h, \phi_{h}^{i}}$ approximate $\mathbf{u}^{i}$ for each $i=0,1, \ldots, n_{0}$ and $p_{h}^{n_{0}} \in Q_{h}$ approximate $p^{n_{0}}$. For each $n=n_{0}, n_{0}+1, \ldots, N-1$, find $\left(\mathbf{u}_{h}^{n+1}, p_{h}^{n+1}\right) \in$ $X_{h, \phi_{h}^{n+1}} \times Q_{h}$ satisfying

$$
\begin{aligned}
& \left(\partial_{\Delta t}^{n+1} \mathbf{u}_{h}, \mathbf{v}_{h}\right)+c_{h}\left(\xi^{n}\left(\mathbf{u}_{h}\right), \mathbf{u}_{h}^{n+1 / 2}, \mathbf{v}_{h}\right) \\
& \quad+\nu\left(\nabla \mathbf{u}_{h}^{n+1 / 2}, \nabla \mathbf{v}_{h}\right)-\left(p_{h}^{n+1 / 2}, \nabla \cdot \mathbf{v}_{h}\right)=\left\langle\mathbf{f}^{n+1 / 2}, \mathbf{v}_{h}\right\rangle, \quad \forall \mathbf{v}_{h} \in X_{h}, \\
& \quad\left(q_{h}, \nabla \cdot \mathbf{u}^{n+1}\right)=0, \quad \forall q_{h} \in Q_{h} .
\end{aligned}
$$

Remark 2.4. Note that $\xi^{n}\left(\mathbf{u}_{h}\right)=\mathbf{u}_{h}^{n+1 / 2}\left(n_{0}=0\right)$ defines the CN-FEM method analyzed e.g. in [19] and $\xi^{n}\left(\mathbf{u}_{h}\right)=\frac{1}{2}\left(3 \mathbf{u}_{h}^{n}-\mathbf{u}_{h}^{n-1}\right)\left(n_{0}=1\right)$ defines the CNLE method e.g. of [2, 15, 25] and $\xi^{n}\left(\mathbf{u}_{h}\right)=2 \mathbf{u}_{h}^{n-1 / 2}-\mathbf{u}_{h}^{n-3 / 2}\left(n_{0}=2\right)$ defines the CNLE(stab) method proposed here.

We now establish energetic stability of BELE and CNLE approximations. We require minimal stability properties of the initial iterates. First define

$$
F_{i c}:=\left\|\mathbf{u}_{h}^{n_{0}}\right\|^{2}+ \begin{cases}\nu \Delta t \sum_{i=0}^{n_{0}}\left|\mathbf{u}_{h}^{i}\right|_{1}^{2}, & \text { if } n_{0} \geq 0 \text { and BELE, } \\ \nu \Delta t \sum_{i=0}^{n_{0}-1}\left|\mathbf{u}_{h}^{i+1 / 2}\right|_{1}^{2}, & \text { if } n_{0} \geq 1 \text { and CNLE. }\end{cases}
$$


The constants $K_{0}$ in Lemma 2.5] and Theorem 2.7 do not depend on a Gronwall constant $\exp (C(T))$. For example,

$$
\begin{aligned}
& K_{0}:=C \nu^{1 / 2} F_{i c}+C\left(\Delta t \sum_{n=n_{0}}^{N-1}\left\|\mathbf{f}^{n+1 / i}\right\|_{-1}^{2}\right)^{1 / 2}+\ldots \\
& \ldots+C \nu^{1 / 2}\left(\Delta t \sum_{n=0}^{N}\left\|E_{h}\left(\phi_{h}^{n+1 / i}\right)\right\|_{1}^{4}\right)^{1 / 2}+\ldots \\
& \ldots+C \nu^{1 / 2}\left(\Delta t \sum_{n=n_{0}}^{N-1}\left\|\partial_{\Delta t} E_{h}\left(\phi_{h}^{n+1 / i}\right)\right\|_{-1}^{2}\right)^{1 / 2}+\ldots \\
& \ldots+C \nu^{3 / 2}\left(\Delta t \sum_{n=n_{0}}^{N-1}\left\|\nabla E_{h}\left(\phi_{h}^{n+1 / i}\right)\right\|^{2}\right)^{1 / 2}+C \nu_{n_{0}+1<n<N-1}\left\|E_{h}\left(\phi_{h}^{n+1 / i}\right)\right\|
\end{aligned}
$$

for some $E_{h}: \Lambda_{h, 0}(\partial \Omega) \rightarrow V_{h, *}$ and $i=1$ for BELE and $i=2$ for CNLE in the proofs of Lemma 2.5 and Theorem 2.7

Lemma 2.5 (BELE solutions are bounded). Fix $\mathbf{f} \in l^{2}\left(W^{-1,2}\right) . \quad$ Fix $\left\{\phi_{h}^{i}\right\}_{i=0}^{N}$ satisfying $\phi_{h} \in l^{4}\left(\Lambda_{h, 0}(\partial \Omega)\right), \partial_{\Delta t} \phi_{h} \in l^{2}\left(\Lambda_{h, 0}(\partial \Omega)\right)$. Suppose further that $\mathbf{u}_{h}^{i} \in$ $V_{h, \phi_{h}^{i}}$ for $i=0,1, \ldots, n_{0}$ so that

$$
F_{i c}<\infty, \quad \text { as } h, \Delta t \rightarrow 0
$$

where $F_{i c}$ is given in (20) and

$$
\left\{\begin{array}{l}
\left|c_{h}\left(\xi^{n}\left(\mathbf{v}_{h}\right), E_{h}\left(\phi_{h}^{n+1}\right), \mathbf{v}_{h}^{n+1}\right)\right| \leq \frac{\nu}{4\left(1+|\mathbf{a}|_{2}^{2}\right)\left(n_{0}+1\right)^{1 / 2}}\left|\xi^{n}\left(\mathbf{v}_{h}\right)\right|_{1}\left|\mathbf{v}_{h}^{n+1}\right|_{1} \\
\forall\left\{\mathbf{v}_{h}^{n}\right\}_{n=0}^{N} \subset V_{h}, \quad \forall n=n_{0}, n_{0}+1, \ldots, N-1
\end{array}\right.
$$

for some extension operator $E_{h}: \Lambda_{h, 0}(\partial \Omega) \rightarrow V_{h, *}$. Then

$$
\left\|\mathbf{u}_{h}\right\|_{l^{\infty}\left(n_{0}+1, N ; L^{2}\right)}+\nu^{1 / 2}\left\|\nabla \mathbf{u}_{h}\right\|_{l^{2}\left(n_{0}+1, N ; L^{2}\right)} \leq \nu^{-1 / 2} K_{0}<\infty
$$

for some $K_{0}>0$.

Proof. See Section 2.2 .

Remark 2.6. Note that $K_{0}<\infty$ uniformly as $h, \Delta t \rightarrow 0$ is ensured, for example, for smooth enough $t \mapsto \phi_{h}(\cdot, t)$ under a small data constraint; i.e., either $\phi_{h}, \nu^{-1}$, or $h$ (at least refined near $\partial \Omega$ where $\phi_{h} \neq 0$ ) is small.

Theorem 2.7 (CNLE solutions are bounded). Fix $\mathbf{f} \in l^{2}\left(W^{-1,2}\right)$. Fix $\left\{\phi_{h}^{i}\right\}_{i=0}^{N}$ satisfying $\phi_{h} \in l^{4}\left(\Lambda_{h, 0}(\partial \Omega)\right), \partial_{\Delta t} \phi_{h} \in l^{2}\left(\Lambda_{h, 0}(\partial \Omega)\right)$. Suppose further that $\mathbf{u}_{h}^{i} \in$ $V_{h, \phi_{h}^{i}}$ for $i=0,1, \ldots, n_{0}$ so that

$$
F_{i c}<\infty, \quad \text { as } h, \Delta t \rightarrow 0
$$

where $F_{i c}$ is given in (20) and

$$
\left\{\begin{array}{l}
\left|c_{h}\left(\xi^{n}\left(\mathbf{v}_{h}\right), E_{h}\left(\phi_{h}^{n+1 / 2}\right), \mathbf{v}_{h}^{n+1 / 2}\right)\right| \leq \frac{\nu}{4\left(1+|\mathbf{a}|_{2}^{2}\right)\left(n_{0}+1\right)^{1 / 2}}\left|\xi^{n}\left(\mathbf{v}_{h}\right)\right|_{1}\left|\mathbf{v}_{h}^{n+1 / 2}\right|_{1}, \\
\forall\left\{\mathbf{v}_{h}^{n}\right\}_{n=0}^{N} \subset V_{h}, \quad \forall n=n_{0}, n_{0}+1, \ldots, N-1
\end{array}\right.
$$


for some extension operator $E_{h}: \Lambda_{h, 0}(\partial \Omega) \rightarrow V_{h, *}$. If $\phi_{h}=0$, then

$$
\left\|\mathbf{u}_{h}\right\|_{l^{\infty}\left(n_{0}+1, N ; L^{2}\right)}+\nu^{1 / 2}\left(\Delta t \sum_{n=n_{0}}^{N-1}\left|\mathbf{u}_{h}^{n+1 / 2}\right|_{1}^{2}\right)^{1 / 2} \leq \nu^{-1 / 2} K_{0}<\infty
$$

where $0<K_{0}<\infty$ is a constant depending on $\left\{\mathbf{u}_{h}^{i}\right\}_{i=0}^{n_{0}}$, f, $\phi_{h}$, but independent of $\nu$. If $\phi_{h} \neq 0$ and

$$
\xi^{n}(\mathbf{u})=b_{0} \mathbf{u}^{n-1 / 2}+b_{1} \mathbf{u}^{n-3 / 2}+\ldots+b_{n_{0}-1} \mathbf{u}^{n-n_{0}+1 / 2},
$$

then CNLE solutions satisfy (23) where $n_{0} \geq 1, a_{0}=b_{0} / 2, a_{i}=\left(b_{i-1}+b_{i}\right) / 2$ for $1 \leq i<n_{0}$, and $a_{n_{0}}=b_{n_{0}-1} / 2$.

Proof. See Section 2.2,

Remark 2.8. As mentioned previously, the result for CNLE for inhomogeneous data with $\xi^{n}(\mathbf{v})=a_{0} \mathbf{v}^{n}+\ldots+\mathbf{v}^{n-n_{0}}$ remains an open question. Of course, $n_{0}=2$ with the alternate extrapolation now refers to a 3 -step extrapolation rather than 2-step to preserve $\mathcal{O}\left(\Delta t^{2}\right)$ accuracy of $\mathrm{CN}$ time-stepping.

2.1. Fundamentals of estimation. The estimates in the following sections are fundamental to our analysis. Let $C>0$ be a generic data-independent constant throughout (depending, possibly on $\Omega$ ). Let $C_{*}>0$ be a generic data-dependent constant (depending, possibly, on $\mathbf{f}, \phi, \mathbf{u}^{0}, \nu^{-1}$ ). In the discrete case, $C, C_{*}$ are independent of $h, \Delta t \rightarrow 0$. The following change of indices formula is required to resolve double sums in stability and convergence analysis of linearly extrapolated BE-FEM and CN-FEM.

Lemma 2.9. Let $\kappa^{n}, \lambda^{n} \in \mathbb{R}$ for all $n \in \mathbb{N}, \alpha^{i} \in \mathbb{R}$ for all $i=0,1, \ldots, n_{0}$. Then,

$$
\sum_{n=n_{0}}^{N-1} \kappa^{n}\left(\sum_{i=0}^{n_{0}} \alpha^{i} \lambda^{n-i}\right)=\sum_{n=0}^{N-1}\left(\sum_{i=i_{0}(n)}^{i_{1}(n)} \alpha^{i} \kappa^{n+i}\right) \lambda^{n}
$$

where

$$
i_{0}(n):=\left\{\begin{array}{ll}
0, & n \geq n_{0}, \\
n_{0}-n, & \text { otherwise },
\end{array} \quad i_{1}(n):= \begin{cases}n_{0}, & n<N-1-n_{0}, \\
N-n, & \text { otherwise }\end{cases}\right.
$$

Proof. Identity (24) follows from a change of indices.

We require Young's inequality in our analysis: for any $a>0, b>0$, and $\delta>0$

$$
a b \leq \frac{1}{q \delta^{q / q^{\prime}}} a^{q}+\frac{\delta}{q^{\prime}} b^{q^{\prime}} .
$$

The following estimate of the explicitly skew-symmetric convective term is obtained through application of Hölder's, Ladyzhenskaya's, and Sobolev embedding inequalities. See [27] for a comprehensive compilation of associated estimates with proof.

Lemma 2.10. Fix $\mathbf{u}, \mathbf{v}, \mathbf{w} \in H^{1}$ and suppose that $\left.(\mathbf{u} \cdot \hat{\mathbf{n}}) \mathbf{v} \cdot \mathbf{w}\right|_{\partial \Omega}=0$. Then

$$
\left|c_{h}(\mathbf{u}, \mathbf{v}, \mathbf{w})\right| \leq C\|\mathbf{u}\|_{1}\|\mathbf{v}\|_{0,3}\|\mathbf{w}\|_{1} .
$$

Energetic stability (which leads to existence) of NS solutions with inhomogeneous data (including general divergence constraint) is investigated in [7, 8, 30, 31]. We conclude without further proof: 
Lemma 2.11 (NSE solutions are bounded). Fix $\phi \in C^{0}\left(H_{0}^{1 / 2}(\partial \Omega)\right)$ and $\mathbf{f} \in$ $L^{2}\left(W^{-1,2}\right)$. Suppose that

$$
4 \nu^{-1}|(\mathbf{w}(\cdot, t) \cdot \nabla \mathbf{w}(\cdot, t), E(\phi(\cdot, t)))| \leq|\mathbf{w}(\cdot, t)|_{1}^{2}, \quad \forall \mathbf{w}(\cdot, t) \in V
$$

is satisfied where $E: H_{0}^{1 / 2}(\partial \Omega) \rightarrow V_{*}$ is an extension operator. Then

$$
\|\mathbf{u}\|_{L^{\infty}\left(L^{2}\right)}+\nu^{1 / 2}\|\mathbf{u}\|_{L^{2}\left(H^{1}\right)} \leq \nu^{-1 / 2} M_{0}
$$

for some $0<M_{0}=M_{0}(\mathbf{f}, \phi)<\infty$ independent of $\nu^{-1}$.

Remark 2.12. Note that for all $\phi \in W^{1, \infty}\left(H_{0}^{1 / 2}(\partial \Omega)\right)$ and for any $\delta>0$ there exists an extension $E_{\delta}: H_{0}^{1 / 2}(\partial \Omega) \rightarrow V_{*}$ that satisfies (27) as long as $\Omega$ is simply connected. Avoiding the smallness constraint on $\phi$ leads to an exponential growth of $\|E(\phi(\cdot, t))\|_{k, p} \leq C \exp (1 / \delta)$ for $k \geq 0, p \geq 1$. Alternatively, we can avoid the smallness assumption on the extension $E(\phi) \in V_{\phi}$ by exploiting the Gronwall Lemma. However, the Gronwall Lemma introduces an exponential dependence of $\mathbf{u}$ on $\nu^{-1}$ that grows as $T \rightarrow \infty$ render such estimates meaningless over long time intervals.

\subsection{Proof of energetic stability.}

Proof of Lemma 2.5. Fix $E_{h}\left(\phi_{h}^{n}\right) \in V_{h, \phi_{h}}$ for each $n \geq 0$. Fix $n \geq n_{0}$. Write $\mathbf{u}_{h}^{n}=\mathbf{w}_{h}^{n}+E_{h}\left(\phi_{h}^{n}\right)$ so that $\mathbf{w}_{h}^{n} \in V_{h}$. Substitute $\mathbf{u}_{h}^{n}=\mathbf{w}_{h}^{n}+E_{h}\left(\phi_{h}^{n}\right)$ into (16) and test with $\mathbf{v}=\mathbf{w}_{h}^{n+1}$. Recall identity (12) so that $c_{h}(\cdot, \mathbf{v}, \mathbf{v})=0$. Then

$$
\begin{aligned}
& \left(\partial_{\Delta t}^{n+1} \mathbf{w}_{h}, \mathbf{w}_{h}^{n+1}\right)+\nu\left|\mathbf{w}_{h}^{n+1}\right|_{1}^{2}=\left(\mathbf{f}^{n+1}, \mathbf{w}_{h}^{n+1}\right)-\left(\partial_{\Delta t}^{n+1} E_{h}\left(\phi_{h}\right), \mathbf{w}_{h}^{n+1}\right) \\
& \quad-\nu\left(\nabla E_{h}\left(\phi_{h}^{n+1}\right), \nabla \mathbf{w}_{h}^{n+1}\right) \\
& \quad-c_{h}\left(\xi^{n}\left(E_{h}\left(\phi_{h}\right)\right), E_{h}\left(\phi_{h}^{n+1}\right), \mathbf{w}_{h}^{n+1}\right)-c_{h}\left(\xi^{n}\left(\mathbf{w}_{h}\right), E_{h}\left(\phi_{h}^{n+1}\right), \mathbf{w}_{h}^{n+1}\right) .
\end{aligned}
$$

Identity $(\mathbf{a}-\mathbf{b}, \mathbf{a})=\frac{1}{2}\left(|\mathbf{a}|^{2}-|\mathbf{b}|^{2}+|\mathbf{a}-\mathbf{b}|^{2}\right)$ gives

$$
\left(\partial_{\Delta t}^{n+1} \mathbf{w}_{h}, \mathbf{w}_{h}^{n+1}\right)=\frac{1}{2 \Delta t}\left(\left\|\mathbf{w}_{h}^{n+1}\right\|^{2}-\left\|\mathbf{w}_{h}^{n}\right\|^{2}\right)+\frac{1}{2 \Delta t}\left\|\mathbf{w}_{h}^{n+1}-\mathbf{w}_{h}^{n}\right\|^{2} .
$$

Apply the duality estimate in $W^{-1,2} \times H_{0}^{1}$ to get

$$
\left(\mathbf{f}^{n+1}, \mathbf{w}_{h}^{n+1}\right)-\left(\partial_{\Delta t}^{n+1} E_{h}\left(\phi_{h}\right), \mathbf{w}_{h}^{n+1}\right) \leq\left(\left\|\mathbf{f}^{n+1}\right\|_{-1}+\left\|\partial_{\Delta t}^{n+1} E_{h}\left(\phi_{h}\right)\right\|_{-1}\right)\left|\mathbf{w}_{h}^{n+1}\right|_{1} .
$$

Apply Cauchy-Schwarz inequality to get

$$
\left|\left(\nabla E_{h}\left(\phi_{h}^{n+1}\right), \nabla \mathbf{w}_{h}^{n+1}\right)\right| \leq\left|E_{h}\left(\phi_{h}^{n+1}\right)\right|_{1}\left|\mathbf{w}_{h}^{n+1}\right|_{1} .
$$

Estimate (26) gives

$$
c_{h}\left(\xi^{n}\left(E_{h}\left(\phi_{h}\right)\right), E_{h}\left(\phi_{h}^{n+1}\right), \mathbf{w}_{h}^{n+1}\right) \leq C\left\|\xi^{n}\left(E_{h}\left(\phi_{h}\right)\right)\right\|{ }_{1}|| E_{h}\left(\phi_{h}^{n+1}\right)||_{0,3}\left|\mathbf{w}_{h}^{n+1}\right|_{1} .
$$

Apply estimates (29), (30), (31), (32) along with (25) to (28) to get

$$
\begin{aligned}
& \frac{1}{2 \Delta t}\left(\left\|\mathbf{w}_{h}^{n+1}\right\|^{2}-\left\|\mathbf{w}_{h}^{n}\right\|^{2}\right)+\frac{1}{2 \Delta t}\left\|\mathbf{w}_{h}^{n+1}-\mathbf{w}_{h}^{n}\right\|^{2}+\nu\left|\mathbf{w}_{h}^{n+1}\right|_{1}^{2} \\
& \leq 5 \nu^{-1}|| \mathbf{f}^{n+1}\left\|_{-1}^{2}+5 \nu^{-1}\right\| \partial_{\Delta t}^{n+1} E_{h}\left(\phi_{h}\right) \|_{-1}^{2}+5 \nu\left|E_{h}\left(\phi_{h}^{n+1}\right)\right|_{1}^{2} \\
& \quad+5 C \nu^{-1}\left\|\xi^{n}\left(E_{h}\left(\phi_{h}\right)\right)\right\|_{1}^{2}\left\|E_{h}\left(\phi_{h}^{n+1}\right)\right\|_{0,3}^{2} \\
& \quad+\frac{\nu}{4}\left|\mathbf{w}_{h}^{n+1}\right|_{1}^{2}-c_{h}\left(\xi^{n}\left(\mathbf{w}_{h}\right), E_{h}\left(\phi_{h}^{n+1}\right), \mathbf{w}_{h}^{n+1}\right) .
\end{aligned}
$$


Young's inequality (25) gives

$$
\left(1+n_{0}\right)^{-1 / 2}\left|\xi^{n}\left(\mathbf{w}_{h}\right)\right|_{1}\left|\mathbf{w}_{h}^{n+1}\right|_{1} \leq \frac{1}{2}\left(\left(1+n_{0}\right)^{-1}\left|\xi^{n}\left(\mathbf{w}_{h}\right)\right|_{1}^{2}+\left|\mathbf{w}_{h}^{n+1}\right|_{1}^{2}\right) .
$$

Apply condition (21) along with (34) to (33). Absorb like terms from right into left-hand sides to get

$$
\begin{aligned}
& \Delta t^{-1}\left(\left\|\mathbf{w}_{h}^{n+1}\right\|^{2}-\left\|\mathbf{w}_{h}^{n}\right\|^{2}\right)+\Delta t^{-1}\left\|\mathbf{w}_{h}^{n+1}-\mathbf{w}_{h}^{n}\right\|^{2} \\
& \quad+\frac{\nu}{2}\left(\left(\frac{3}{2}-\frac{1}{2\left(1+|\mathbf{a}|_{2}^{2}\right)}\right)\left|\mathbf{w}_{h}^{n+1}\right|_{1}^{2}-\left(\frac{1}{2\left(1+|\mathbf{a}|_{2}^{2}\right)\left(1+n_{0}\right)}\right)\left|\xi^{n}\left(\mathbf{w}_{h}\right)\right|_{1}^{2}\right) \\
& \quad \leq 5 \nu^{-1}\left\|\mathbf{f}^{n+1}\right\|_{-1}^{2}+5 \nu^{-1}\left\|\partial_{\Delta t}^{n+1} E_{h}\left(\phi_{h}\right)\right\|_{-1}^{2}+5 \nu\left|E_{h}\left(\phi_{h}^{n+1}\right)\right|_{1}^{2} \\
& \quad+5 C \nu^{-1}\left\|\xi^{n}\left(E_{h}\left(\phi_{h}\right)\right)\right\|_{1}^{2}\left\|E_{h}\left(\phi_{h}^{n+1}\right)\right\|_{0,3}^{2} .
\end{aligned}
$$

From the change of indices identity (24), we obtain

$$
\begin{aligned}
\sum_{n=n_{0}}^{N-1}\left|\xi^{n}\left(\mathbf{w}_{h}\right)\right|_{1}^{2} & \leq \sum_{n=n_{0}}^{N-1} \sum_{i=0}^{n_{0}}\left(1+n_{0}\right)\left|a_{i}\right|^{2}\left|\mathbf{w}_{h}^{n-i}\right|_{1}^{2} \\
& =\left(1+n_{0}\right) \sum_{n=0}^{N-1}\left|\mathbf{w}_{h}^{n}\right|_{1}^{2} \sum_{i=i_{0}(n)}^{i_{1}(n)}\left|a_{i}\right|^{2} \leq\left(1+n_{0}\right)|\mathbf{a}|_{2}^{2} \sum_{n=0}^{N-1}\left|\mathbf{w}_{h}^{n}\right|_{1}^{2}
\end{aligned}
$$

so that

$$
\begin{aligned}
& \left(\frac{3}{2}-\frac{1}{2\left(1+|\mathbf{a}|_{2}^{2}\right)}\right) \sum_{n=n_{0}}^{N-1}\left|\mathbf{w}_{h}^{n+1}\right|_{1}^{2}-\left(\frac{1}{2\left(1+|\mathbf{a}|_{2}^{2}\right)\left(1+n_{0}\right)}\right) \sum_{n=n_{0}}^{N-1}\left|\xi^{n}\left(\mathbf{w}_{h}\right)\right|_{1}^{2} \\
& \geq\left(\frac{3}{2}-\frac{1}{2\left(1+|\mathbf{a}|_{2}^{2}\right)}\right) \sum_{n=n_{0}}^{N-1}\left|\mathbf{w}_{h}^{n+1}\right|_{1}^{2}-\frac{|\mathbf{a}|_{2}^{2}}{2\left(1+|\mathbf{a}|_{2}^{2}\right)} \sum_{n=0}^{N-1}\left|\mathbf{w}_{h}^{n}\right|_{1}^{2} \\
& \geq \sum_{n=n_{0}+1}^{N}\left|\mathbf{w}_{h}^{n}\right|_{1}^{2}-\frac{|\mathbf{a}|_{2}^{2}}{2\left(1+|\mathbf{a}|_{2}^{2}\right)} \sum_{i=0}^{n_{0}}\left|\mathbf{w}_{h}^{i}\right|_{1}^{2} .
\end{aligned}
$$

Apply Young's inequality and Sobolev imbedding to show

$$
\sum_{n=n_{0}}^{N-1}\left\|\xi^{n}\left(E_{h}\left(\phi_{h}\right)\right)\right\|_{1}^{2}\left\|E_{h}\left(\phi_{h}^{n+1}\right)\right\|_{0,3}^{2} \leq C \sum_{n=0}^{N}\left\|E_{h}\left(\phi_{h}^{n}\right)\right\|_{1}^{4} .
$$

Sum from $n=n_{0}$ to $n=N-1$ in (35). Apply (36) and simplify to get

$$
\begin{aligned}
& \left\|\mathbf{w}_{h}^{N}\right\|^{2}+\sum_{n=n_{0}}^{N-1}\left\|\mathbf{w}_{h}^{n+1}-\mathbf{w}_{h}^{n}\right\|^{2}+\nu \Delta t \sum_{n=n_{0}}^{N-1}\left|\mathbf{w}_{h}^{n+1}\right|_{1}^{2} \\
& \leq\left\|\mathbf{w}_{h}^{n_{0}}\right\|^{2}+\frac{\nu}{2} \Delta t \sum_{n=0}^{n_{0}}\left|\mathbf{w}_{h}^{n}\right|_{1}^{2}+C \nu^{-1} \Delta t \sum_{n=0}^{N}\left\|E_{h}\left(\phi_{h}^{n}\right)\right\|_{1}^{4} \\
& \quad+5 \nu^{-1} \Delta t \sum_{n=n_{0}}^{N-1}\left(\left\|\mathbf{f}^{n+1}\right\|_{-1}^{2}+\left\|\partial_{\Delta t}^{n+1} E_{h}\left(\phi_{h}^{n+1}\right)\right\|_{-1}^{2}+\nu^{2}\left|E_{h}\left(\phi_{h}^{n+1}\right)\right|_{1}^{2}\right)
\end{aligned}
$$


Apply the triangle inequality with $\mathbf{u}_{h}^{n}=\mathbf{w}_{h}^{n}-E_{h}\left(\phi_{h}^{n}\right)$ and (37) to get

$$
\begin{aligned}
\nu \| & \nabla \mathbf{u}_{h}\left\|_{l^{2}\left(n_{0}+1, N ; L^{2}\right)}^{2} \leq\right\| \mathbf{u}_{h}^{n_{0}}\left\|^{2}+\nu\right\| \nabla \mathbf{u}_{h} \|_{l^{2}\left(0, n_{0} ; L^{2}\right)}^{2} \\
& +\nu\left\|\nabla E_{h}\left(\phi_{h}\right)\right\|_{l^{2}\left(0, n_{0} ; L^{2}\right)}^{2}+C \nu^{-1}\left\|E_{h}\left(\phi_{h}\right)\right\|_{l^{4}\left(H^{1}\right)}^{4} \\
& +C \nu^{-1}\|\mathbf{f}\|_{l^{2}\left(n_{0}+1, N ; W^{-1,2}\right)}^{2}+C \nu^{-1}\left\|\partial_{\Delta t}^{n+1} E_{h}\left(\phi_{h}\right)\right\|_{l^{2}\left(n_{0}+1, N ; W^{-1,2}\right)}^{2} \\
& +C \nu\left\|\nabla E_{h}\left(\phi_{h}\right)\right\|_{l^{2}\left(n_{0}+1, N ; L^{2}\right)}^{2}
\end{aligned}
$$

and

$$
\begin{aligned}
& \left\|\mathbf{u}_{h}^{n}\right\|_{l^{\infty}\left(n_{0}+1, N ; L^{2}\right)}^{2} \leq\left\|\mathbf{u}_{h}^{n_{0}}\right\|^{2}+\nu\left\|\nabla \mathbf{u}_{h}\right\|_{l^{2}\left(0, n_{0} ; L^{2}\right)}^{2} \\
& \quad+\nu\left\|\nabla E_{h}\left(\phi_{h}\right)\right\|_{l^{2}\left(0, n_{0} ; L^{2}\right)}^{2}+C \nu^{-1}\left\|E_{h}\left(\phi_{h}\right)\right\|_{l^{4}\left(H^{1}\right)}^{4} \\
& \quad+C\left\|E_{h}\left(\phi_{h}\right)\right\|_{l^{\infty}\left(n_{0}+1, N ; L^{2}\right)}^{2}+C \nu^{-1}\|\mathbf{f}\|_{l^{2}\left(n_{0}+1, N ; W^{-1,2}\right)}^{2} \\
& \quad+C \nu^{-1}\left\|\partial_{\Delta t}^{n+1} E_{h}\left(\phi_{h}\right)\right\|_{l^{2}\left(n_{0}+1, N ; W^{-1,2}\right)}^{2}+C \nu\left\|\nabla E_{h}\left(\phi_{h}\right)\right\|_{l^{2}\left(n_{0}+1, N ; L^{2}\right)}^{2}
\end{aligned}
$$

The estimate (22) follows from (38), (39) under the assumed regularity.

Proof of Theorem 2.7. The proof of Theorem 2.7 follows the proof in Lemma 2.5 closely. For CN-FEM, test with $\mathbf{v}_{h}=\mathbf{w}^{n+1 / 2}$ to get

$$
\begin{aligned}
& \frac{1}{2 \Delta t}\left(\left\|\mathbf{w}_{h}^{n+1}\right\|^{2}-\left\|\mathbf{w}_{h}^{n}\right\|^{2}\right)+\nu\left|\mathbf{w}_{h}^{n+1 / 2}\right|_{1}^{2} \\
& =\left(\mathbf{f}^{n+1}, \mathbf{w}_{h}^{n+1}\right)-\left(\partial_{\Delta t}^{n+1} E_{h}\left(\phi_{h}\right), \mathbf{w}_{h}^{n+1 / 2}\right)-\nu\left(\nabla E_{h}\left(\phi_{h}^{n+1 / 2}\right), \nabla \mathbf{w}_{h}^{n+1 / 2}\right) \\
& \quad-c_{h}\left(\xi^{n}\left(E_{h}\left(\phi_{h}\right)\right), E_{h}\left(\phi_{h}^{n+1 / 2}\right), \mathbf{w}_{h}^{n+1 / 2}\right)-c_{h}\left(\xi^{n}\left(\mathbf{w}_{h}\right), E_{h}\left(\phi_{h}^{n+1 / 2}\right), \mathbf{w}_{h}^{n+1 / 2}\right)
\end{aligned}
$$

instead of (28). The remaining estimates are obtained similar to those in the proof of Lemma 2.5. The main difference, aside from exchanging indices $n+1$ with $n+1 / 2$, concerns the legitimacy of estimate (36) in the case of CNLE. When $\phi_{h}=0$, there is no problem because there is no contribution from the nonlinearity. However, for general $\phi_{h} \neq 0$, we require the prescribed form of the linearization $\xi^{n}(\mathbf{u})=b_{0} \mathbf{u}^{n-1 / 2}+b_{1} \mathbf{u}^{n-3 / 2}+\ldots+b_{n_{0}-1} \mathbf{u}^{n-n_{0}+1 / 2}$ which allows the nonlinearity to be absorbed in a similar way as shown in (36) for BELE. Proceeding as before, we prove (23).

\section{NumericAL INVESTIGATION}

In this section we investigate how CNLE(stab), with the alternate extrapolation

$$
\xi^{n}(\mathbf{u})=2 \mathbf{u}^{n-1 / 2}-\mathbf{u}^{n-3 / 2},
$$

improves flow statistics and preserves flow integrity relative to CNLE with conventional extrapolation $\xi^{n}(\mathbf{u})=\frac{3}{2} \mathbf{u}^{n}-\frac{1}{2} \mathbf{u}^{n-1}$. The energy dissipation rate is given by

$$
\varepsilon(t):=\nu|\mathbf{u}(\cdot, t)|_{1}^{2} .
$$

In the previous discussion, our work suggests that CNLE solutions might have worse control on the size of $\varepsilon(t)$ than CNLE(stab). To be precise, we compare herein the 
size of the numerical dissipation rate $\varepsilon_{c n l e}^{n}$ for CNLE and CNLE(stab) applied to flow past a $2 \mathrm{~d}$ cylinder where

$$
\varepsilon_{\text {cnle }}^{n+1 / 2}:=\nu\left|\mathbf{u}_{h}^{n+1 / 2}\right|_{1}^{2} .
$$

For the problem setup, consider the channel $([0,2.2] \times[0,0.41])-\Omega_{s}$ where $\Omega_{s}$ is circular obstacle with diameter $=0.1$ centered at $(0.2,0.2)$. The flow has boundary conditions:

$$
\begin{aligned}
& \mathbf{u}(x, y=0)=\mathbf{u}(x, y=0.41)=\left.\mathbf{u}\right|_{\partial \Omega_{s}}=0 \\
& \mathbf{u}(x=0, y)=\mathbf{u}(x=2.2, y)=\frac{4}{0.41^{2}} y(0.41-y)
\end{aligned}
$$

Let the initial data $\left(\mathbf{u}^{0}, p^{0}\right)$ satisfy the (steady) Stokes problem. For high enough Reynolds number (albeit below turbulence levels) vortices will begin shedding in the wake of $\Omega_{s}$ at a regular frequency (von Kármán vortex street). This is a similar experiment performed in [23], but there with time-dependent boundary conditions and starting from rest.

We compare 3 approximate NSE flows obtained with CN-FEM, CNLE, and the newly proposed CNLE(stab). We solve each problem on the time interval $[0,15]$ with Taylor-Hood finite elements on the same mesh. The mesh is generated by Delaunay-Voronoi triangulation in FreeFem ++ and contains 143100 velocity degrees of freedom (161168 total degrees of freedom) with 128 vertices on $\partial \Omega_{s}$. For CN-FEM, we resolve the nonlinearity with Newton iterations so that the $H^{1}$ residual error is less than $10^{-12}$ at each time step. For CNLE and CNLE(stab), the iterates $\mathbf{u}_{h}^{i}$ for $i=1, \ldots, n_{0}$ are obtained with a fixed point nonlinear iteration so that the $H^{1}$ residual error is less than $10^{-12}$.
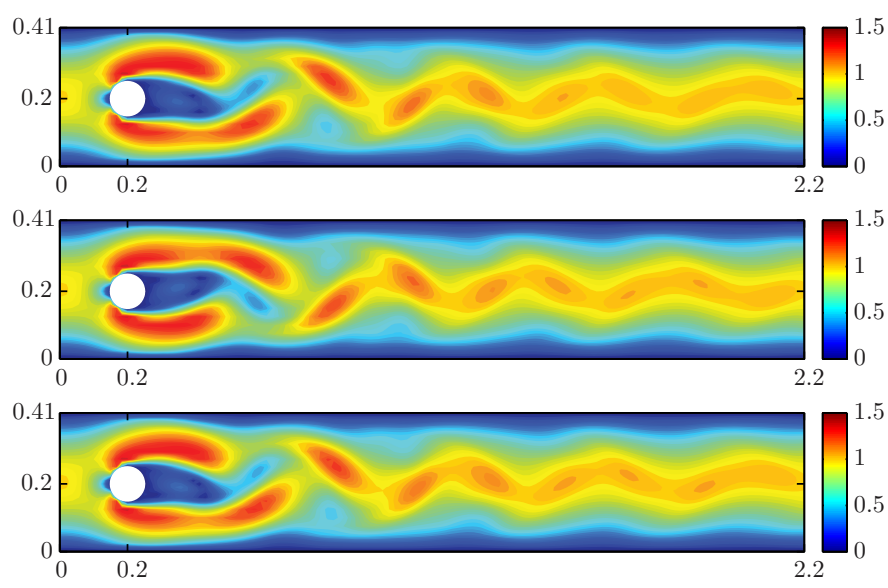

Figure 1. Flow past cylinder: magnitude of velocity field computed with CN-FEM (newton) at (top) $T=5$, (middle) $T=10$, (bottom) $T=15$ with $\Delta t=0.005$. Notice the distinct and periodic vortex shedding associated with the von Kàrmàn vortex street. 

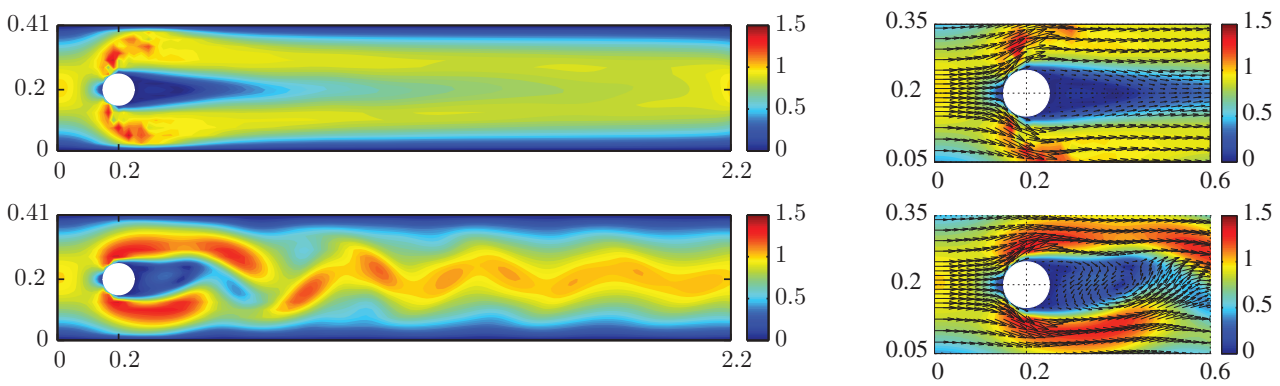

Figure 2. Flow past cylinder at $T=10$ with $\Delta t=0.002$ : speed-profile and velocity field for (top) CNLE and (bottom) CNLE(stab). Notice that CNLE suppresses all vortex shedding predicted by CNLE(stab).

We present the magnitude of the velocity field of the CN-FEM flow for $\nu^{-1}=$ 1000 computed with $\Delta t=0.005$ at $T=5,10,15$ in Figure 1, The characteristic vortex shedding off the back of the cylinder is realized here. We present the magnitude of the velocity field and vector field of the CNLE and CNLE(stab) flow for the same conditions at $T=10$ computed with $\Delta t=0.005$ in Figure 2. In this case the CNLE(stab) method closely models the flow generated by CN-FEM, but the CNLE method is over-diffused and fails to capture the expected vortex shedding.

The degradation of CNLE flow approximation is clearly seen in the plots displayed in Figures 3, 4. In each plot, we plot a statistic measuring the numerical energy dissipation rate $\varepsilon_{c n l e}^{n+1 / 2}$ over the time interval $[0,15]$ for $\nu^{-1}=400,600,800$, 1000, 1200, 1400. In Figure 3 we measure the maximum $\varepsilon_{c n l e}^{n+1 / 2}$ on the time interval and in Figure 4 we measure the $l^{2}(0, T)$-norm of $\varepsilon_{c n l e}^{n+1 / 2}$. The curve on each plot for $\mathrm{CN}-\mathrm{FEM}$ is the bottom-most curve and decreases as $\nu^{-1}$ as expected. The curve for CNLE(stab) matches CN-FEM when $\Delta t=0.001$, but deviates slightly starting at $\nu^{-1}=1200$ when $\Delta t=0.002$. Conversely, the curve for CNLE deviates from CN-FEM starting at $\nu^{-1}=1400$ when $\Delta t=0.001$, and deviates more significantly starting at $\nu^{-1}=600$ when $\Delta t=0.002$.

In Figures 5 and 6 we present the behavior of an alternate measure of the numerical dissipation based on $\varepsilon_{c n l e}^{n}$ rather than the average $\mathbf{u}^{n+1 / 2}$ natural for the $\mathrm{CN}$ method. Interestingly, the curves for CN-FEM and CNLE(stab) are comparable for $\varepsilon_{c n l e}^{n+1 / 2}$ and $\varepsilon_{c n l e}^{n}$ but the curve for CNLE deviates from the expectation even more dramatically for $\varepsilon_{c n l e}^{n}$.

In Figure 7 we plot $\varepsilon_{c n l e}^{n}$ for CN-FEM $(\Delta t=0.005)$, CNLE $(\Delta t=0.002)$, and $\mathrm{CNLE}(\mathrm{stab})(\Delta t=0.002)$ respectively for $\nu^{-1}=600,800,1000$ with respect to the numerical time levels over $[0,15]$. The curves for CN-FEM and CNLE(stab) match closely with a relative decrease between each curve with increasing $\nu^{-1}$. Conversely, the curves for CNLE increases with $\nu^{-1}$. 

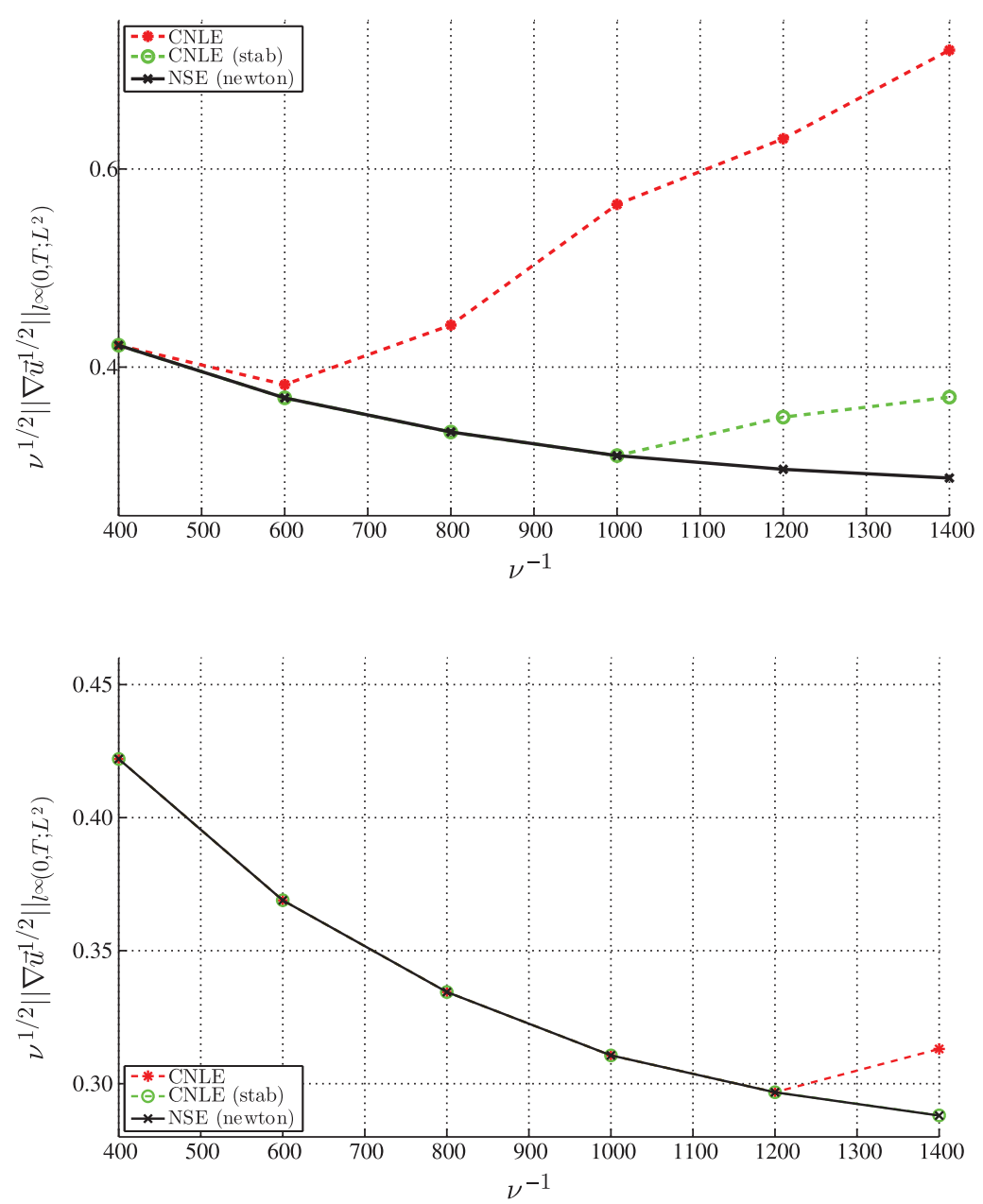

Figure 3. Flow past cylinder: maximal energy dissipation rate at $t^{n+1 / 2}$ vs. $\nu^{-1}$ for CN-FEM solutions computed with $\Delta t=0.005$ and CNLE, CNLE(stab) solutions with (top) $\Delta t=0.002$, and (bottom) $\Delta t=0.001$. 

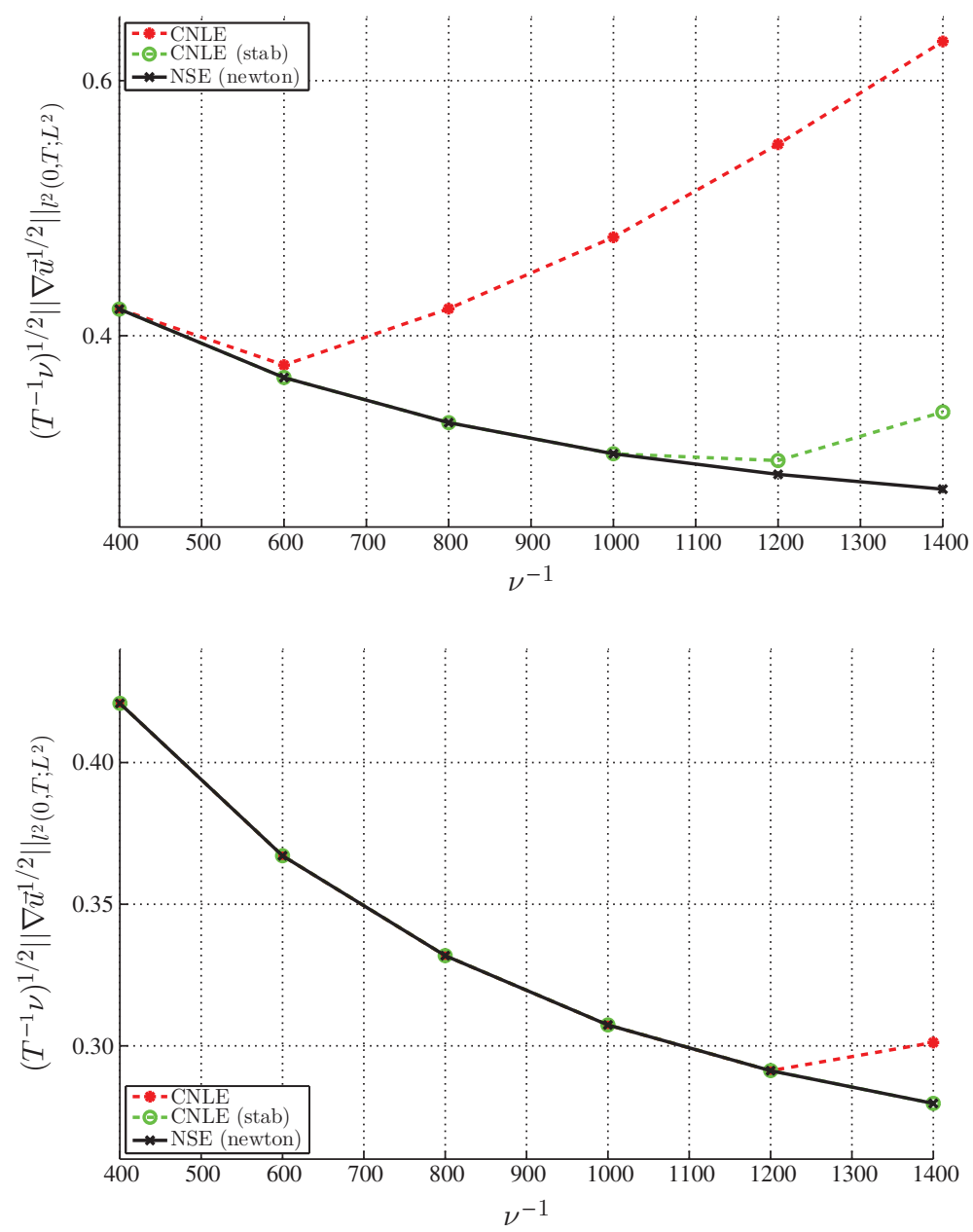

Figure 4. Flow past cylinder: time-averaged energy dissipation rate at $t^{n+1 / 2}$ vs. $\nu^{-1}$ for $\mathrm{CN}-\mathrm{FEM}$ solutions computed with $\Delta t=$ 0.005 and CNLE, CNLE(stab) solutions with (top) $\Delta t=0.002$, and (bottom) $\Delta t=0.001$. 

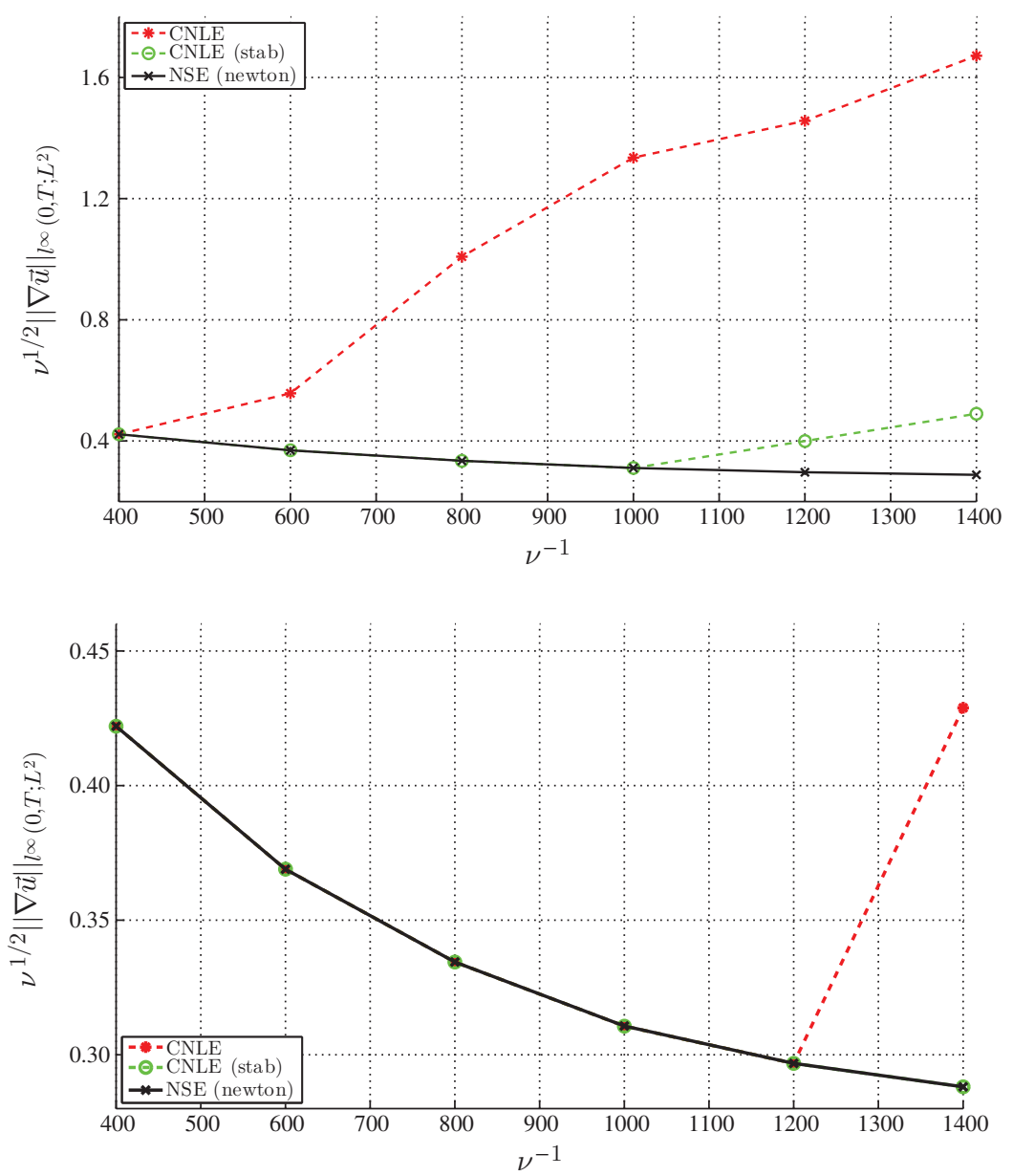

FIgURE 5. Flow past cylinder: maximal energy dissipation rate vs. $\nu^{-1}$ for CN-FEM solutions computed with $\Delta t=0.005$ and CNLE, CNLE(stab) solutions with (top) $\Delta t=0.002$, and (bottom) $\Delta t=$ 0.001 . 

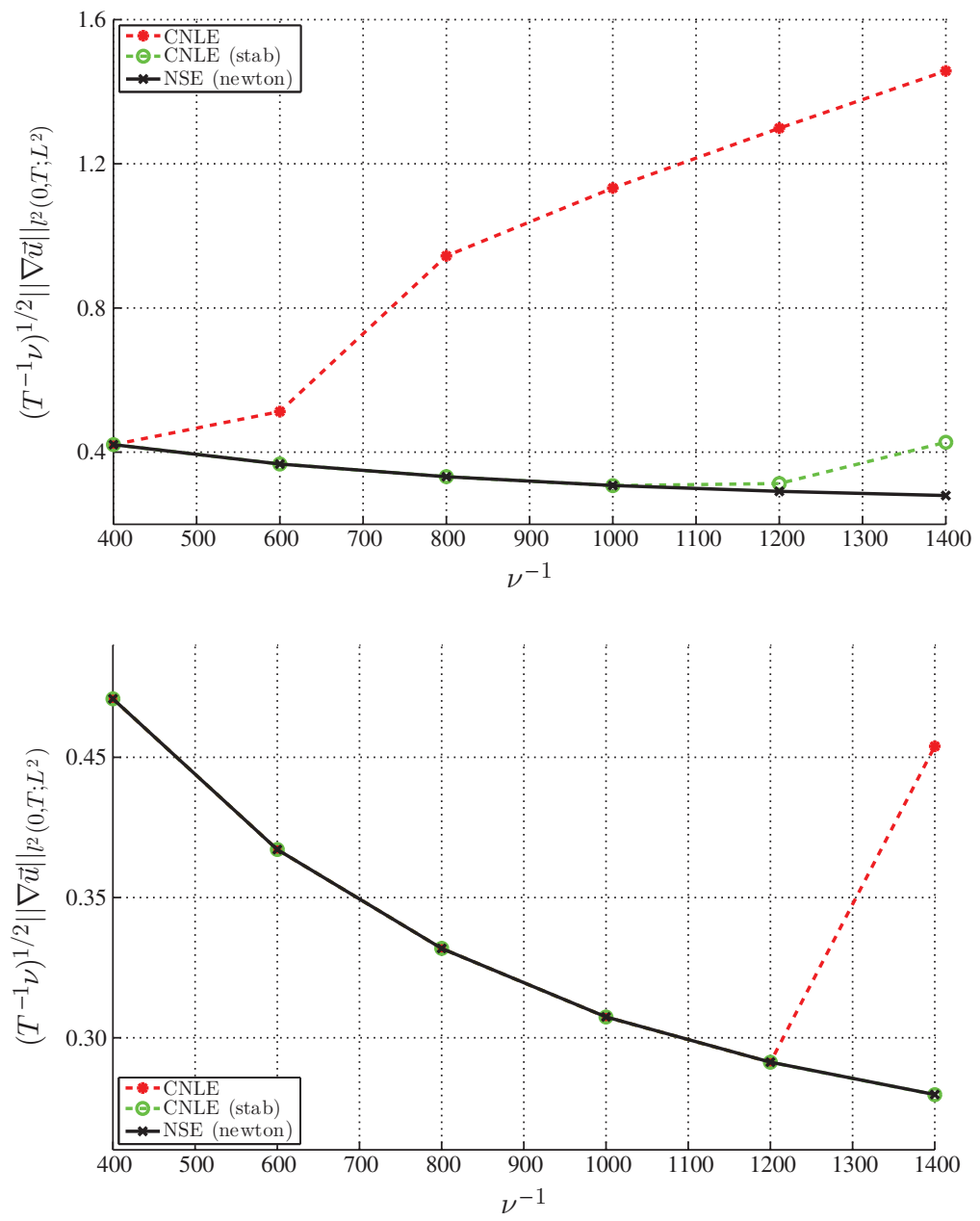

Figure 6. Flow past cylinder: time-averaged energy dissipation rate vs. $\nu^{-1}$ for CN-FEM solutions computed with $\Delta t=0.005$ and CNLE, CNLE(stab) solutions with (top) $\Delta t=0.002$, and (bottom) $\Delta t=0.001$. 

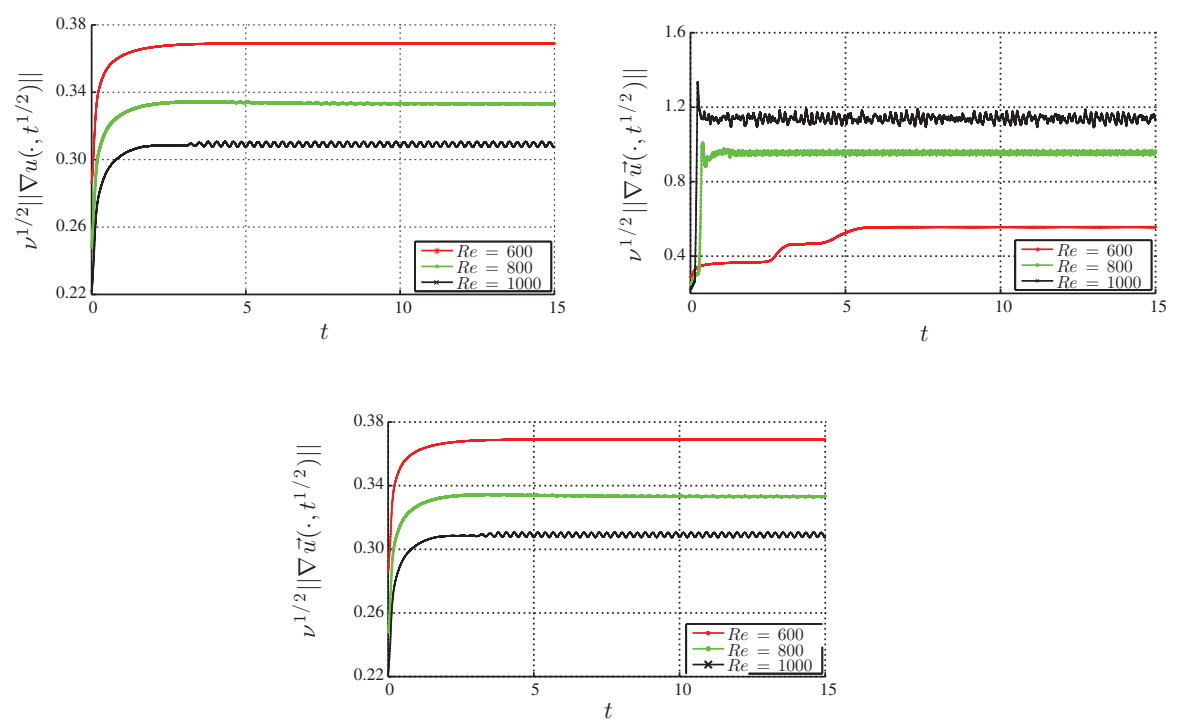

FiguRE 7. Flow past cylinder: energy dissipation rate vs. time for (top-left) CN-FEM with $\Delta t=0.005$, (top-right) CNLE with $\Delta t=0.002$, (bottom) CNLE(stab) with $\Delta t=0.002$. Notice that the CN-FEM and CNLE(stab) curves demonstrate the expected relative decrease in energy dissipation with increasing $\nu^{-1}$ unlike conventional CNLE.

\section{Conclusions}

We investigated herein the stability and accuracy of an extrapolated CrankNicolson time-stepping method for a finite element spatial discretization of the NSE. We propose a novel, nonstandard linear extrapolation of the convecting velocity that encourages speed-up from solving the fully nonlinear $\mathrm{CN}$ scheme denoted by CNLE(stab). We prove that CNLE(stab) is energetically stable without a Gronwall exponential factor (this result is not achievable under standard techniques for the inhomogeneous Dirichlet problem for conventional CNLE). The numerical results in Section 3 confirm that CNLE(stab) is clearly advantageous relative to conventional CNLE.

\section{REFERENCES}

1. G. Apte, S. Canstantinescu, F. Ham, G. Iaccarino, and P. Mahesh, K. Moin, Large-eddy simulation of reacting turbulent flows in complex geometries, J. Appl. Mech. 73 (2006), 364371 .

2. G.A. Baker, Galerkin approximations for the Navier-Stokes equations, Tech. report, Havard University, 1976.

3. G.A. Baker, V. Dougalis, and O. Karakashian, On a higher order accurate, fully discrete galerkin approximation to the Navier-Stokes equations, Math. Comp. 39 (1982), 339-375. MR669634 (84h:65096) 
4. J. H. Bramble, J. E. Pasciak, and P. S. Vassilevski, Computational scales of Sobolev norms with application to preconditioning, Math. Comp. 69 (1999), no. 230, 69-463. MR.1651742 (2000k:65088)

5. S.C. Brenner and L.R. Scott, The Mathematical Theory of Finite Element Methods, second ed., Springer, Berlin, 2002. MR.1894376 (2003a:65103)

6. Y. Duan, W. Wang, and X. Yang, The approximation of a Crank-Nicolson scheme for the stochastic Navier-Stokes equations, J. Comput. Appl. Math. 225 (2009), 31-43. MR2490168 (2010h:65009)

7. R. Farwig, H. Kozono, and H. Sohr, Global weak solutions of the Navier-Stokes equations with nonhomogeneous boundary data and divergence, Rend. Semin. Ma. Univ. P. 125 (2010), 51-70.

8. A.V. Fursikov, M.D. Gunzburger, and L.S. Hou, Inhomogeneous boundary value problems for the three-dimensional evolutionary Navier-Stokes equations, J. Math. Fluid Mech. 4 (2002), 45-75. MR:1891074(2002m:35181)

9. G.P. Galdi, An Introduction to the Mathematical Theory of the Navier-Stokes Equations, vol. I, Springer-Verlag, New York, 1994. MR1284205 (95i:35216a)

10. V. Girault and J.-L. Lions, Two-grid finite-element schemes for the transient Navier-Stokes problem, Model. Math. Anal. Numer. 35 (2001), no. 5, 945-980. MR1866277 (2003a:76078)

11. V. Girault and P.A. Raviart, Finite Element Approximations of the Navier-Stokes Equations, Lecture Notes in Mathematics, Springer-Verlag, New York, 1979. MR548867 (83b:65122)

12. _ Finite Element Methods for Navier-Stokes Equations, Springer-Verlag, Berlin, 1986. MR851383(88b:65129)

13. P. M. Gresho, R. L. Sani, and M. S. Engelman, Incompressible Flow and the Finite Element Method, John Wiley \& Sons, LTD, New York, 1998.

14. M. D. Gunzburger and J. S. Peterson, On conforming finite element methods for the inhomogeneous stationary Navier-Stokes equations, Numer. Math. 42 (1983), 173-194. MR720658 (85f:76050)

15. Y. He, Two-level method based on finite element and Crank-Nicolson extrapolation for the time-dependent Navier-Stokes equations, SIAM J. Numer. Anal. 41 (2003), 1263-1285. MR2034880(2004k:65173)

16. Y. He and W. Sun, Stability and convergence of the Crank-Nicolson/Adams-Bashforth scheme for the time-dependent Navier-Stokes equations, Math. Comput. 76 (2007), no. 257, 115-136. MR2261014(2007j:76105)

17. for the time-dependent Navier-Stokes equations, Math. Comput. 76 (2007), no. 257, 115-136. MR2261014(2007j:76105)

18. J.G Heywood and R. Rannacher, Finite element approximation of the nonstationary NavierStokes problem, II. Stability of solutions and error estimates uniform in time, SIAM J. Numer. Anal. 23 (1986), 750-777. MR 849281 (88b:65132)

19. , Finite element approximation of the nonstationary Navier-Stokes problem, IV. Error analysis for second-order time discretization, SIAM J. Numer. Anal. 19 (1990), 275-311. MR650052 (83d:65260)

20. Y. Hou and Q. Liu, A two-level finite element method for the Navier-Stokes equations based on a new projection, Applied Mathematical Modelling 34 (2010), 383-399. MR2556189 (2011c:65216)

21. Y. Huang and M. Mu, An alternating Crank-Nicolson method for decoupling the GinzburgLandau equations, SIAM J. Numer. Anal. 35 (1998), no. 5, 1740-1761. MR.1640013 (99m:65215)

22. V. John, A comparison of parallel solvers for the incompressible Navier-Stokes equations, Computing and Visualization in Science 1 (1999), no. 4, 193-200.

23. _ Reference values for drag and lift of a two-dimensional time-dependent flow around a cylinder, Int. J. Numer. Meth. Fl. 44 (2004), 777-788.

24. V. John, M. Gunar, and J. Rang, A comparison of time-discretization/linearization approaches for the incompressible Navier-Stokes equations, Comput. Methods Appl. Mech. Engrg. 195 (2006), 5995-6010. MR2250930 (2007b:76094)

25. A. Labovsky, W. Layton, C. Manica, M. Neda, and L. Rebholz, The stabilized, extrapolated trapezoidal finite element method for the Navier-Stokes equations, Comput. Methods Appl. Mech. Eng. 198 (2009), 958-974. MR2498863(2010i:65211) 
26. W. Layton, A two-level discretization method for the Navier-Stokes equations, Comput. Math Appl. 26 (1993), 33-38. MR1220955 (94i:65127)

27. W. Layton and L. Tobiska, A two-level method with backtracking for the Navier-Stokes equations, SIAM J. Numer. Anal. 35 (1998), 2035-2054. MR1639994 (99g:65115)

28. K. Matsuzaki, M. Munekata, H. Ohba, and Ushijima, Numerical study on particle motions in swirling flows in a cylinder separator, J. Therm. Sci. 15 (2006), no. 2, 181-186.

29. M.R. Ohm, H.Y. Lee, and J.Y. Shin, $L^{2}$-error estimates of the extrapolated Crank-Nicolson discontinuous Galerkin approximations for nonlinear Sobolev equations, J. Inequal. Appl. 2010 (2010), 1-17. MR2600188(2011c:65221)

30. J.-P. Raymond, Stokes and Navier-Stokes equations with nonhomogeneous boundary conditions, Anal. Non Linéaire 24 (2007), 921-951. MR 2371113 (2009a:35188)

31. _ Stokes and Navier-Stokes equations with a nonhomogeneous divergence condition, Discret. Contin. Dyn. S. B 14 (2010), no. 4, 1537-1564. MR2679654 (2011g:35323)

32. L. R. Scott and S. Zhang, Finite element interpolation of nonsmooth functions satisfying boundary conditions, Math. Comp. 54 (1990), no. 190, 483-493. MR1011446 (90j:65021)

33. J.N. Sorensen and W.Z Shen, Numerical modeling of wind turbine wakes, J. Fluids Eng. 124 (2002), 393-399.

34. M. Tabata and D. Tagami, Error estimates for finite element approximations of drag and lift in nonstationary Navier-Stokes flows, Japan J. Indust. Appli. Math. 17 (2000), 371-389. MR 1794176 (2002j:65095)

University of Pittsburgh, 615 Thackeray Hall, Pittsburgh Pennsylvania 15260 Current address: 2259 Shady Avenue, Pittsburgh, Pennsylvania 15217

E-mail address: rni1@psualum.com

$U R L:$ http://www.math.pitt.edu/ ${ }^{\sim} \operatorname{rni1}$ 